Kong. Res. J. 3(1) : 6-11, 2016

ISSN 2349-2694

Kongunadu Arts and Science College, Coimbatore.

\title{
VIRTUAL SCREENING OF GINKGO BILOBA FOR THERAPEUTIC POTENTIALS AGAINST PARKINSON'S DISEASE
}

\author{
Kavitha, V., S. Jone Kirubavathy, M.S. Sivaramkumar and R. Velmurugan* \\ Department of Chemistry, Kongunadu Arts and Science College, Coimbatore- 641029. \\ *E.mail: velmuruganchemist57@gmail.com
}

ABSTRACT

Parkinson's disease (PD) is a neurodegenerative disorder that affects $2 \%$ of the population older than 60 years. Monoamine Oxidase B (MAO-B) inhibitors improve the symptoms of Parkinson's disease and can delay the progress. Inhibition of MAO-B, further prevent breakdown of dopamine in the brain and reduce the motor symptoms associated with PD. Ginkgo biloba has a number of therapeutic properties and contains phytonutrients that helps in improvement of neurological disorders. In present study, phytonutrients of Ginkgo biloba namely Myricetin, Quercetin, Isorhamnetin, Kaempferol, Ginkgolides A-C, and Ginkgolide J were selected for Molecular docking against Monoamine Oxidase-B enzyme. The Molecular Docking studies were performed using Autodock 4.2 and interaction between MAO-B and compounds were analyzed. The efficiency of the compound was screened based on the binding energy existing between the protein and inhibitor. The docking studies show that the phytochemicals of Ginkgo biloba against MAO-B were quite effective. The potential compound can be subjected to further clinical trials and can be an alternative in the future treatment of Parkinson's disease.

Keywords: Virtual Screening, Molecular Docking, Auto Dock, Ginkgo biloba, Phytonutrients.

\section{INTRODUCTION}

The age dependent neurodegenerative diseases include Parkinson's disease and Alzheimer's disease (Arumo et al., 2003), which are caused by genetic and environmental influences (Jenner and Olanow, 1998) and lead to the accumulation of protein aggregation thereby causing oxidative stress and inflammation (Behl, 1999). Abnormal action of the monoamine oxidase $B$ isoform has been associated with neurological dysfunctions including parkinson's disorder and alzheimer's disorder whereas the monoamine oxidase A isoform seems to be associated with psychiatric considerations including depression and cardiac cellular degeneration (Bortolato et al., 2008). MAO-B inhibitors are used for the treatment of Parkinson's disease and for symptoms associated with Alzheimer's disease (Binda et al., 2004; Terud and Langston, 1989). In the present work, our purpose was to distinguish correct poses of inhibitor in the binding pocket of monoamine oxidase $\mathrm{B}$ and to predict the affinity between the inhibitor and monoamine oxidase $\mathrm{B}$.

In other words, in this study docking procedure describes a process by which two molecules fit together in three-dimensional space (Kitchen et al., 2004). Computer aided drug design is an applicable method that can study these interactions and describe significant characteristics for monoamine oxidase binding site recognition (Delogu et al., 2011; Harkcom and Bevan, 2007). Extracts of Ginkgo biloba leaves produce reversible inhibition of rat brain monoamine oxidase (MAO) (White et al., 1996). Mao inhibition was due to the phytonutrients present in Ginkgo biloba. The computer aided drug design is an attempt to study the interaction of phytonutrients with MAO-B which in turn helps to treat the symptoms of Parkinson's disease.

The use of computers to predict the binding of libraries of small molecules to known target structures is an increasingly important component in the drug discovery process (Schoichet, 2004; Koppen, 2009 ). There is a wide range of software packages available for the conduct of molecular docking simulations like, Auto Dock, GOLD, and FlexX (Collignon et al., 2011). Auto Dock 4.2 is the most recent version which has been widely used for virtual screening, due to its enhanced docking speed (Dykstra, 2007). Docking is applied to predict the binding orientation of small molecular drug candidates to protein targets, subsequently predicting the affinity and activity of the drug candidates (Goodsell, 2009; Morris et al., 2009). Docking is often applied to predict binding affinities of drug candidates in virtual screening experiments and in considering structure-activity relationships to prioritize synthesis of new drugs (Wu et al., 2003). The present study deals with the examination of the 
interactions between potentials from Ginkgo biloba and MAO-B protein by molecular docking method in order to calculate the minimum binding energy (kcal/mol) between them. Molecular docking determines the binding affinity between the protein and ligands which aims to determine the 3D conformation and binding interactions.

\section{MATERIALS AND METHODS}

\subsection{Protein structure}

The high-resolution crystal structure of monoamine oxidase- $B$, co-crystalized with its irreversible inhibitor 6-hydroxy-N-propargyl-1(R)aminoindan, was obtained from the Protein Data Bank (PDB entry code $1 \mathrm{~S} 3 \mathrm{E}, 1.6 \mathrm{~A}^{\circ}$ resolution). The study was carried out on only one subunit of the enzyme protein (Yelekçi et al., 2007) shown in Figure 1. The water molecules were removed during modeling. The energy minimized protein structure was included prior to docking to accommodate hydrogen atoms.

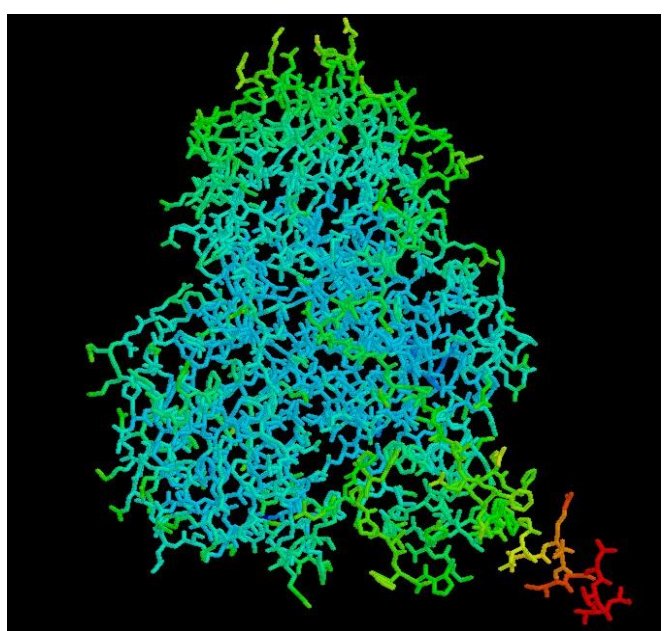

Fig. 1. 3D structure of Monoamine oxidase - B molecule (PDB ID: 1S3E)

\subsection{Phytochemicals}

The structures of phytochemicals namely Myricetin, Quercetin (Oyama et al., 1994), Ginkgolide A, Ginkgolide B, Ginkgolide C, Ginkgolide J, Bilobalide (Teris, 2002), kaempferol, Isorhamnetin (Xu et al., 2012) used in this study were retrieved from Pubchem compound database. The 2D structures of molecules were converted to 3D structures using Open Babel software (O'Boyle et al., 2011). These phytochemicals satisfied Lipinski's rule of 5 and ADME properties.

\subsection{Binding site Prediction}

The binding site in MAO-B was determined using Computer Atlas of Surface Topology of
Proteins (CASTp) (Dundas et al., 2006). CASTp helps in identifying the geometric properties of protein pockets which are assumable positions on protein surface. The residues within the binding site were identified. Potential active site of protein calculated by CASTp in Fig. 2. Showed there are several pockets which fit in the role of active site.

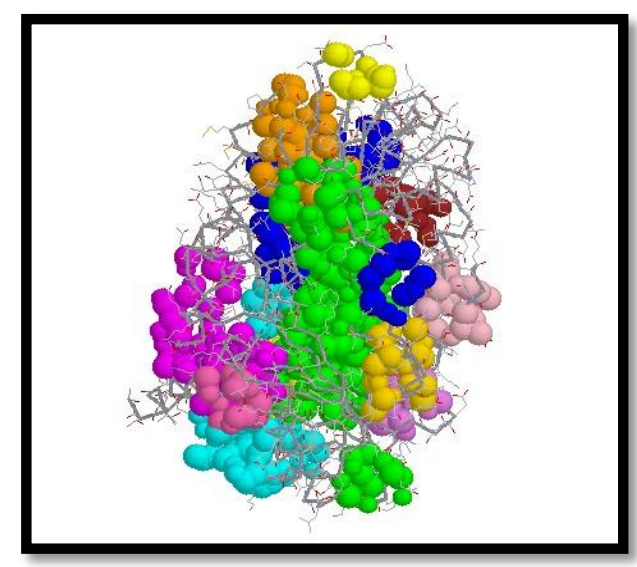

Fig. 2. Active sites predicted in the MAO-B using CASTp server

\subsection{Molecular docking}

Molecular docking combined with a scoring function can be used to screen potential drugs insilico to identify molecules that are likely to bind to protein target of interest. To perform the docking model, the Auto Dock 4.2 suite molecular-docking tool was used and the methodology was followed (Gowthaman et al., 2008). AutoDock was employed to perform a docking simulation using a Lamarckian genetic algorithm (Morris et al., 1998). Auto Dock 4.0 is widely distributed molecular docking software which performs the flexible docking of the ligands into a known protein structure. The default parameters of the automatic settings were used. Each docking experiment consisted of 10 docking runs with 150 individuals and 500,000 energy evaluations. The size of the grid box is key parameter in Auto Dock. The volume of the box was fixed to $27000 \AA$ to have large search space. The Auto Dock results indicated the binding position and bound conformation of the protein, as well as hydrogen bond interactions between the protein and ligand molecule. The docked conformation which had the minimum binding energy was selected to analyze the mode of binding.

\section{RESULTS AND DISCUSSION}

The phytochemicals were docked using Auto dock 4.2 successfully. The interactions and binding energy of the phytochemicals are listed in Table 1. Good interactions were observed between the amino 
acid residues of the protein and phytochemical molecules. The phytochemicals showed binding energy between -7.78 to $-8.70 \mathrm{kcal} / \mathrm{mol}$. The results were analyzed based on the binding energy of the complex. The number of $\mathrm{H}$-bonds was calculated between atoms of protein-ligand docked complex. Quercetin and Kaempferol illustrate high affinity for the protein molecules with score of -8.70 and -8.68 shown in Fig. 3 and 4. Quercetin and Kaempferol showed six and five hydrogen bond interactions with MAO-B respectively. Quercetin illustrated interactions with GLN206, GLY434, SER59, TYR435 and TYR60 residues of the protein. Kaempferol showed five hydrogen bond interactions with SER59, TYR60, TYR188, and GLY434 residues of the MAO-B protein and bound to the active sites. The compounds were bound to the active site of the MAO-B receptor.

Table 1. Docking results of Ginkgo biloba phytochemicals with MAO-B protein.

\begin{tabular}{|c|c|c|c|c|}
\hline Phytochemicals & Compound structure & $\begin{array}{l}\text { Binding } \\
\text { energy } \\
\text { kcal/mol }\end{array}$ & Hbonds & $\begin{array}{l}\text { Residues interacting } \\
\text { with ligand (Hbonds) }\end{array}$ \\
\hline Ginkgolide A & & -8.33 & 2 & SER59, LYS296 \\
\hline Ginkgolide B & & -8.65 & 4 & $\begin{array}{l}\text { SER59, LYS296, } \\
\text { GLN206, TYR398 }\end{array}$ \\
\hline Ginkgolide C & & -7.78 & 3 & $\begin{array}{l}\text { SER59, } \\
\text { TYR398 }\end{array}$ \\
\hline Ginkgolide J & & -8.57 & 3 & $\begin{array}{l}\text { LYS296, } \\
\text { TYR60 }\end{array}$ \\
\hline
\end{tabular}




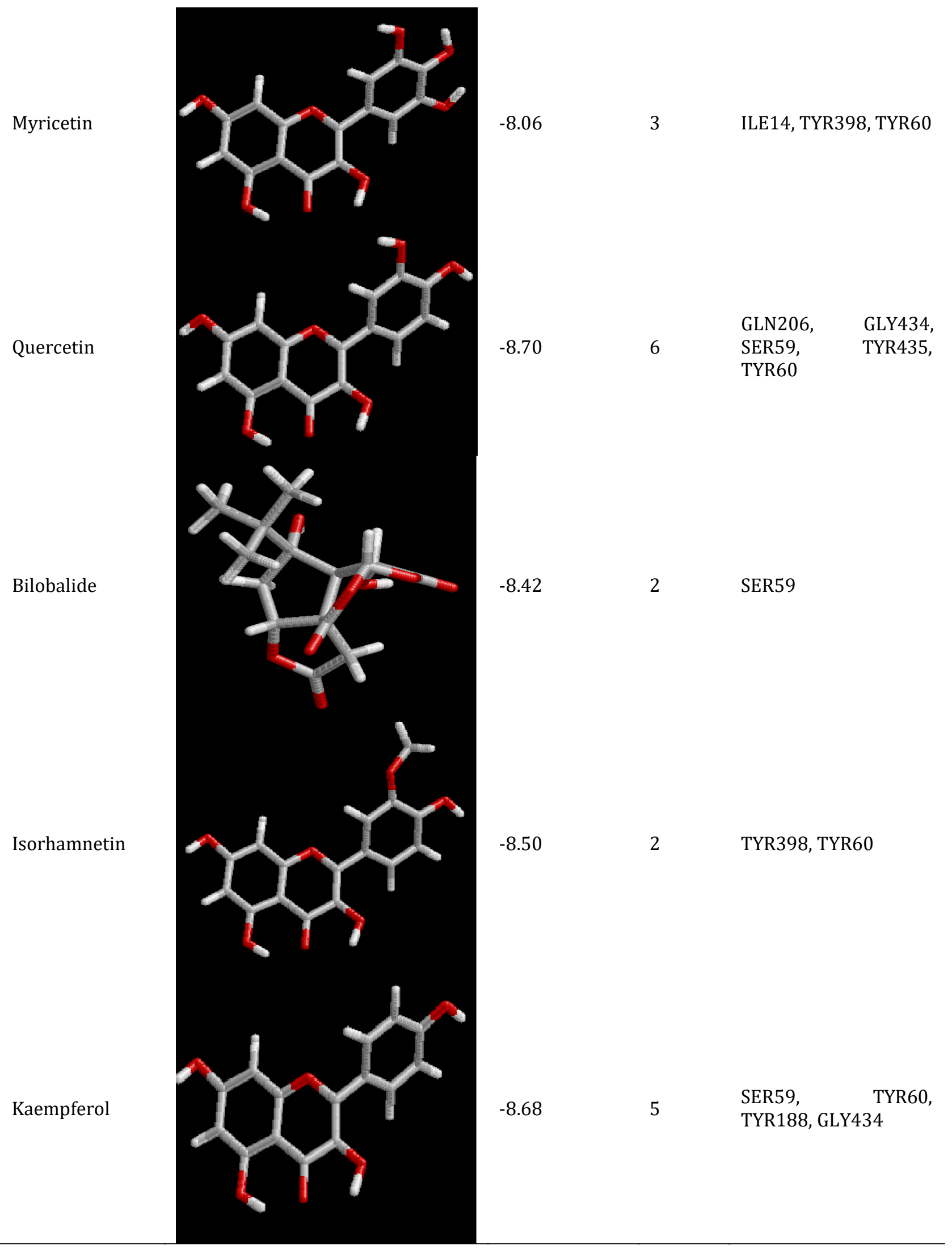




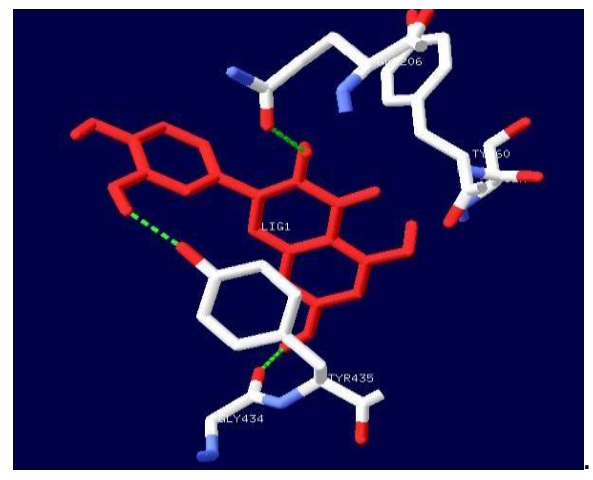

Fig. 3a. Quercetin interacting with residues of MAO-B protein

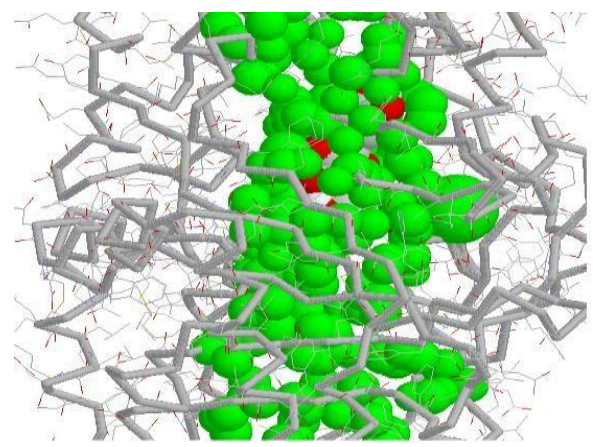

Fig. 3b. Quercetin bound to the active site of protein molecule.

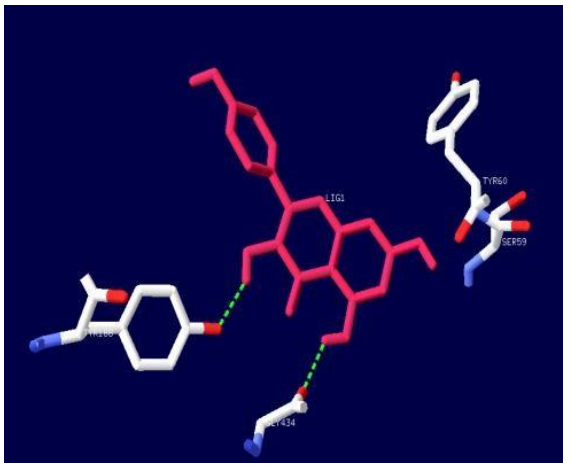

Fig. 4a. Kaempferol interacting with residues of MAO-B protein.

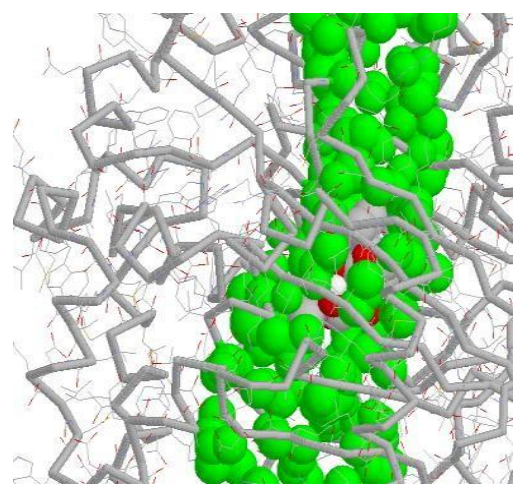

Fig. 4b. Kaempferol bound to the active site of protein molecule.
The screening of phytochemicals from Ginkgo biloba against MAO-B is carried out using molecular docking methods. Screening of Phytonutrients compounds showed the binding affinity towards MAO-B receptor. The Quercetin and Kaempferol were screened with least binding energy of -8.70 and -8.68 and were selected as Lead molecule. The molecular docking of the two compounds showed the binding mode and interaction energy. $\mathrm{H}$-bond pattern was analyzed and confirmed the inhibition of MAO -B target and show the molecular activity of phytochemicals. This work based on Insilico studies, concluded that Quercetin and Kaempferol possess better activity against MAOB. Further In vivo studies on these compounds can be done to confirm the inhibition and used in the treatment of Parkinson's disease.

\section{REFERENCES}

Aruoma, O.I., T. Bahorun and L.S. Jen, (2003). Neuroprotection by bioactive components in medicinal and food plant extracts. Mutation Res. 544: 203-215

Behl, C. (1999). Alzheimer's disease and oxidative stress: Implications for novel therapeutic approaches. Prog. Neurobiol. 57: 301-323

Binda, C., F. Hubalek, M. Li, D.E. Edmondson and A. Mattevi, (2004). Crystal structure of human monoamine oxidase $\mathrm{B}$, a drug target enzyme monotopically inserted into the mitochondrial outer membrane. FEBS Lett. 564: 225-228

Bortolato, M., K. Chen and J.C. Shih, (2008). Monoamine oxidase inactivation: from pathophysiology to therapeutics. Adv. Drug Deliv. Rev. 60: 1527-1533.

Delogu, G., C. Picciau, G. Ferino, E. Quezada, G. Podda, E. Uriarte and D. Vina, (2011). Synthesis, human monoamine oxidase inhibitory activity and molecular docking studies of 3heteroarylcoumarin derivatives. Eur. J. Med. Chem. 46: 1147-1152.

Goodsell, D.S. (2009). Computational docking of biomolecular complexes with AutoDock. Cold Spring Harbor protocols 2009:pdb prot5200.

Gowthaman, U., M. Jayakanthan and D. Sundar, (2008). Molecular docking studies of dithionitrobenzoic acid and its related compounds to protein disulfide isomerase: Computational screening of inhibitors to HIV, 1 entry. BMC Bioinformatics 9:S12-S14. 
Harkcom, W.T. and D.R. Bevan, (2007). Molecular docking of inhibitors into monoamine oxidase B. Biochem. Biophy. Res. Comm. 360:401-406.

Jenner, P. and C.W. Olanow, (1998). Understanding cell death in Parkinson's disease. Ann. Neurol. 3(1):72-84.

Joe Dundas, Zheng Ouyang, Jeffery Tseng, Andrew Binkowski, Yaron Turpaz, and Jie Liang 2006. CASTp: computed atlas of surface topography of proteins with structural and topographical mapping of functionally annotated residues. Nucleic Acid Res. 34:W116-W118.

Kitchen, D.B., H.L.N. Decornez, J.R. Furr and J.R. Bajorath, (2004). Docking and scoring in virtual screening for drug discovery: methods and applications. Nat. Rev. Drug Dis. 3: 935-949.

Koppen, H. (2009). Virtual screening - what does it give us? Curr. Opin. Drug Disc. Dev. 12: 397-407.

Morris, G.M., D.S. Goodsell, R.S. Halliday, R. Huey, W.E. Hart, R.K. Belew and A.J. Olson, (1998). Automated docking using a Lamarckian genetic algorithm and empirical binding free energy function. J. Comp. Chem. 19:1639-1662

Morris, G.M., R. Huey, W. Lindstrom, M.F. Sanner, R.K. Belew, D.S. Goodsell and A.J. Olson, (2009). AutoDock4 and AutoDockTools4: Automated docking with selective receptor flexibility. J. Comp. Chem. 30: 2785-2791.

O'Boyle, N.M., M. Banck, C.A. James, C. Morley, T. Vandermeersch and G.R. Hutchison, (2011). Open Babel: An open chemical toolbox. J. Chem. Inform. 3: 33.

Schoichet, B.K. (2004). Virtual screening of chemical libraries. Nature 43: 862-865.
Teris A van Beek, (2002). Chemical analysis of Ginkgo biloba leaves and extracts. $J$. Chromatography 967(1): 21-55,

Tetrud, J.W. and J.M. Langston, (1989). The effect of deprenyl (selegiline) on the natural history of Parkinson's disease. Science 245: 519-522

White, H.L., P.W. Scates and B.R. Cooper, (1996). Extracts of Ginkgo biloba leaves inhibit monoamine oxidase. Life Sci. 58(16): 13151321.

Wu, G., D.H. Robertson, C.L. Brooks and M. Vieth, (2003). Detailed analysis of grid based molecular docking: A case study of C-DOCKER. A CHARM based MD docking algorithm. J. Comp. Chem. 24:1549-1562.

Xu, S.L., R.C. Choi, K.Y. Zhu, K.W. Leung, A.J. Guo, D. Bi, H. Xu, D.T. Lau, T.T. Dong, K.W. Tsim and Isorhamnetin, (2012). A Flavonol Aglycone from Ginkgo biloba L., Induces Neuronal Differentiation of Cultured PC12 Cells: Potentiating the Effect of Nerve Growth Factor. Evid. Based Comp. Alternat. Med. 278273.

Yasuo Oyama, A. Paul, Fuchs, Norihiro Katayama and Katsuhiko Noda, (1994). Myricetin and quercetin, the flavonoid constituents of Ginkgo biloba extract, greatly reduce oxidative metabolism in both resting and $\mathrm{Ca}^{2+}$-loaded brain neurons. Brain Res. 635(1-2): 125-129.

Yelekçi, K., O. Karahan and M. Toprakçi, (2007). Docking of novel reversible monoamine oxidase-B inhibitors: efficient prediction of ligand binding sites and estimation of inhibitors thermodynamic properties. J. Neural Transm. 114(6): 725-32. 